\title{
SOME INFINITE FIBONACCI GROUPS
}

\author{
by D. L. JOHNSON \\ (Received 12th February 1974)
}

The Fibonacci groups are a special case of the following class of groups first studied by G. A. Miller (7). Given a natural number $n$, let $\theta$ be the automorphism of the free group $F=\left\langle x_{1}, \ldots, x_{n} \mid\right\rangle$ of rank $n$ which permutes the subscripts of the generators in accordance with the cycle $(1,2, \ldots, n)$. Given a word $w$ in $F$, let $R$ be the smallest normal subgroup of $F$ which contains $w$ and is closed under $\theta$. Then define $G_{n}(w)=F / R$ and write $A_{n}(w)$ for the derived factor group of $G_{n}(w)$. Putting, for $r \geqq 2, k \geqq 1$,

$$
w=x_{1} \ldots x_{r} x_{r+k}^{-1}
$$

with subscripts reduced modulo $n$, we obtain the groups $F(r, n, k)$ studied in (1) and (2), while the $F(r, n, 1)$ are the ordinary Fibonacci groups $F(r, n)$ of (3), (5) and (6). To conform with earlier notation, we write $A(r, n, k)$ and $A(r, n)$ for the derived factor groups of $F(r, n, k)$, and $F(r, n)$ respectively.

Our purpose in the present note is threefold: firstly to explain the connection between the two apparently different formulae for $|A(r, n)|$ given in (5) (numbered (3) and (6)), secondly to derive necessary and sufficient conditions for $A_{n}(w)$ to be infinite, and finally to apply this to the groups $A(r, n, k)$ by way of example. The second item is the analogue of a result of Dunwoody (4), while the third extends Theorems 6 and 7 of (1) and at the same time complements Theorem 1 of (2).

The symbol | . | will stand indiscriminately for the order of an element of a group, the order of a group or the absolute value of a complex number, it being clear from the context which is intended. $Z[x]$ denotes the ring of polynomials over the integers $\mathbf{Z}$, while $\mathbf{Z}\left\langle x \mid x^{n}\right\rangle$ is the integral group ring of a cyclic group of order $n$, so that

$$
Z\left\langle x \mid x^{n}\right\rangle \cong Z[x] /\left(x^{n}-1\right),
$$

as rings. Finally, we observe the usual conventions with regard to empty sums (for example, formula (1) below when $s=0$ ) and empty products (for example, the definition of $f * g$ when $f$ is a constant polynomial).

The $n$-generator, $n$-relation presentation given above for $F(r, n)$ shows that a relation matrix for $A(r, n)$ is the circulant matrix $C$ whose first row is

where,

$$
\underbrace{k+1, \ldots, k+1,}_{s} k-1, \underbrace{k, \ldots, k}_{n-s-1}
$$

$$
r=k n+s, \quad 0 \leqq s<n \text {. }
$$


Thus we obtain formula (6) of (5), viz.

$$
|A(r, n)|= \pm \operatorname{det} C= \pm \prod_{i=1}^{n}\left(\sum_{j=1}^{s}(k+1) \omega_{i}^{j-1}+(k-1) \omega_{i}^{s}+\sum_{j=s+2}^{n} k \omega_{i}^{j-1}\right) \text {, }
$$

where $\omega_{1}, \ldots, \omega_{n}$ are distinct $n$th roots of unity. We can express this more conveniently as follows: let $b_{i-1}$ be the exponent-sum of $x_{i}$ in $w=x_{1} \ldots x_{r} x_{r+1}^{-1}$ and put

Writing

$$
f(x)=b_{0}+b_{1} x+\ldots+b_{n-1} x^{n-1} .
$$

formula (1) reduces to

$$
g(x)=x^{n}-1,
$$

$$
|A(r, n)|=\prod_{g(\xi)=0}|f(\xi)| .
$$

The presentation for $F(r, n)$ on $r$ generators $\left(x_{1}, \ldots, x_{r}\right)$ obtained using Tietze transformations in the obvious way, leads to the relation matrix

$$
M^{n}-I
$$

for $A(r, n)$, where $M$ is the companion matrix of the polynomial

$$
f^{\prime}(x)=x^{r}-x^{r-1}-\ldots-x-1 .
$$

Thus we have another expression for $|A(r, n)|$ :

$$
|A(r, n)|=\prod_{i=1}^{n}\left|\xi_{i}^{n}-1\right|,
$$

where $\xi_{1}, \ldots, \xi_{\text {r }}$ are the zeros of $f^{\prime}(x)$, in other words

Now we have

$$
|A(r, n)|=\prod_{f^{\prime}(\xi)=0}|g(\xi)| .
$$

$-f^{\prime}(x)=\left(1+x^{n}+\ldots+x^{(k-1) n}\right)\left(1+x+\ldots+x^{n-1}\right)+x^{k n}\left(1+x+\ldots+x^{s-1}-x^{5}\right)$, so that

$$
f+f^{\prime} \equiv 0(\bmod (g))
$$

Definition. Let $f, g \in \mathbf{Z}[x]$ and define $f * g \in \mathbf{Z}$ to be the product of the values of $g$ on the complex zeros of $f$.

The following easily-proved theorem now shows that (2) and (3) are in fact the same formula.

Theorem 1. For polynomials $f, g, f^{\prime} \in \mathrm{Z}[x]$, we have

(i) $f * g= \pm g * f$, if $f$ and $g$ are monic,

(ii) $g * f=g * f^{\prime}$, if $f \equiv f^{\prime}(\bmod g)$,

(iii) $g * f f^{\prime}=(g * f)\left(g * f^{\prime}\right)$.

Passing now to the $G_{n}(w)$, let $b_{i-1}$ be the exponent sum of $x_{i}$ in $w$ and define

$$
f(x)=b_{0}+b_{1} x+\ldots+b_{n-1} x^{n-1},
$$

as above, a member of $Z[x]$. Call $f(x)$ the polynomial associated with $w$. 
Dunwoody's Theorem (4) asserts that $G_{n}(w)$ is perfect if and only if the polynomial associated with $w$ is a unit in $Z\left\langle x \mid x^{n}\right\rangle$. Our next result is an analogue of this.

Theorem 2. Let $f(x)$ be the polynomial associated with the word $w$ in the free group of rank $n$, and let $g(x)=x^{n}-1$. The following three assertions are then equivalent:

(a) $G_{n}(w)$ has an infinite abelian factor group,

(b) $g * f=0$,

(c) $f(x)$ is a zero-divisor in $\mathbf{Z}\left\langle x \mid x^{n}\right\rangle$.

Proof. We first prove the equivalence of $(a)$ and $(b) .(a)$ is equivalent to the assertion that $A_{n}(w)$ is infinite. Now a relation matrix for $A_{n}(w)$ is the circulant matrix $C$ with first row $\left(b_{0}, b_{1}, \ldots, b_{n-1}\right)$, and so $A_{n}(w)$ is infinite if and only if $\operatorname{det} C=0$. But $\operatorname{det} C= \pm g * f$, so the result follows. To show that $(b)$ and (c) are equivalent, first note that $g * f=0$ if and only if $g$ and $f$ have a common zero, that is $f(\xi)=0$ for some $n$th root $\xi$ of unity. Let $\phi_{k}(x)$ be the cyclotomic polynomial of order $k ;(b)$ is equivalent to the assertion: $\phi_{k}(x)$ is a divisor of $f(x)$ for some $k$ dividing $n$. Since $\phi_{k}(x) \phi_{k}^{\prime}(x)=x^{n}-1$ for some $\phi_{k}^{\prime}(x) \in \mathbf{Z}[x]$, this condition is clearly equivalent to $(c)$.

Thus we see that $A_{n}(w)$ is infinite if and only if the polynomial associated with $w$ vanishes on some $n$th root of unity. We apply this to the classification of the infinite members of the set $A(r, n, k)$, thereby generalising Theorems 6 and 7 of (1), as well as Corollary 2 of (5). Theorem 1 of (2) asserts that $F(r, n, k)$ is metacyclic of order $r^{n}-1$ provided that

$$
r \equiv 1(\bmod n) \text { and }(n, k)=1 .
$$

Our theorem shows that $\boldsymbol{F}(r, n, k)$ is infinite if

$$
r \equiv 1(\bmod n) \text { and }(n, k) \neq 1 .
$$

Theorem 3. $A(r, n, k)$ is infinite if and only if at least one of the following two conditions holds:

(i) $(r-1, k, n) \neq 1$,

(ii) $v_{2}(r+1)$ and $v_{2}(n)$ are each greater than $v_{2}(k-1)$,

where $v_{2}$ denotes the 2-part of a positive integer and $v_{2}(0)=\infty$.

Proof. By Theorem 2, $A(r, n, k)$ is infinite if and only if the polynomial

$$
1+x+\ldots+x^{r-1}-x^{r+k-1}
$$

vanishes on an $n$th root of unity. Since $r \geqq 2$, this is equivalent to the vanishing of

$$
\left(x^{r+k}-x^{r+k-1}\right)-\left(x^{r}-1\right)
$$

on some non-trivial $n$th root of unity. We first assume that some $n$th root $\xi$ of unity is a root of (5), so that

$$
\xi^{r+k}-\xi^{r+k-1}=\xi^{r}-1
$$

E.M.S. $-19 / 3-X$ 
It follows that

so that either

$$
|\xi-1|=\left|\xi^{r}-1\right|,
$$

$$
\xi^{r}=\xi \text { or } \xi^{r}=\xi^{-1} \text {. }
$$

In the first case, substitution in (6) yields

so that

$$
\xi^{k}(\xi-1)=(\xi-1),
$$

$$
\xi \neq 1, \xi^{k}=1, \quad \xi^{r-1}=1, \quad \xi^{n}=1,
$$

from which (i) follows. In the second case, substitution in (6) yields

$$
\xi^{k-1}-\xi^{k-2}=\xi^{-1}-1,
$$

and multiplication of this by $\xi /(\xi-1)$ gives

The conditions

$$
\xi^{k-1}=-1 .
$$

now give condition (ii).

$$
\xi \neq 1, \xi^{k-1}=-1, \xi^{r+1}=1, \xi^{n}=1
$$

For the converse, let $h \neq 1$ be a common factor of $r-1, k$ and $n$. If $\xi$ is a primitive $h$ th root of unity, then $\xi \neq 1, \xi^{n}=1$ and (5) vanishes on $\xi$. If, on the other hand, (ii) holds, let $h=2 \cdot v_{2}(k-1)$, so that $h$ is a divisor both of $r+1$ and $n$, and $h \neq 1$. Again let $\xi$ be a primitive $h$ th root of unity, so that again $\xi \neq 1, \xi^{n}=1$. Since $\xi^{2(k-1)}=1$ and $\xi^{k-1} \neq 1$, we must have $\xi^{k-1}=-1$ and $\xi$ is again a zero of (5), which completes the proof.

\section{REFERENCES}

(1) C. M. Campbell and E. F. Robertson, A note on a class of generalized Fibonacci groups (to appear).

(2) C. M. CAMPBell and E. F. RoBertson, The orders of certain metacyclic groups, Bull. London Math. Soc. (to appear).

(3) J. H. Conway, Solution to Advanced Problem 5327 Amer. Math. Monthly, 74 (1967), 91-93.

(4) M. J. Dunwoody, Cyclic presentations and 3-manifolds (to appear).

(5) D. L. Johnson, A note on the Fibonacci groups, Israel J. Math. (to appear).

(ด) D. L. Johnson, J. W. WAMsLeY and D. WRIGHT, The Fibonacci groups, Proc. London Math. Soc. (to appear).

(7) G. A. Miller, Groups generated by $n$ operators each of which is the product of the $\mathrm{n}-1$ remaining ones, Amer.J. Math. 30 (1908), 93-98.

\section{UNIVERSITY OF NOTTINGHAM}

